\title{
Effect of Intravenous Lignocaine on the Sevoflurane Requirements Monitored by the Bispectral Index
}

\author{
Dr. B. Velmurugan M.D Da ${ }^{1}$, Dr. Aishwarya ${ }^{2}$ \\ ${ }^{1}$ Senior Assistant Professor, Institute of Anesthesiology, Madurai medical college, Madurai \\ ${ }^{2}$ Post Graduate , Institute of Anesthesiology, Madurai medical college, Madurai
}

\begin{abstract}
Inadequate general anesthesia results in intraoperative awareness with or without recall and the consequent postoperative morbidity. Bi Spectral Index (BIS) helps the anesthesiologist in assessing the conscious state of the patient and in modifying the dose of anesthetic drugs to maintain an adequate depth of anesthesia. Systemic lidocaine has been used in several studies to know its benefits on the outcome of anesthesia. Having inflammatory-modulating property, it significantly reduces pain and allows rapid discharge. We planned a study to evaluate the effect of intravenous lidocaine in reducing sevoflurane requirements during maintenance of general anesthesia Methods randomized double-blinded controlled trial enrolling 60 patients in the study. Informed written consent was obtained from all the patients and they were then randomly allocated into two groups of 30 each, namely Group L (lignocaine)and Group C (control).BIS monitor Pulseoximetry NIBP Capnography (intraoperatively), ECG were measured in both groups Results Study showed that the amount of sevoflurane infused in the lidocaine group, to maintain BIS between 40 and 60 was significantly reduced, and also the intraoperative fentanyl requirements were significantly lower in the lidocaine group. It also significantly maintains the hemodynamic stability throughout the procedure and reduces the intraoperative analgesic requirements in patients undergoing laparoscopic surgeries.
\end{abstract}

Keywords: Anesthesia, Lidocaine, Bispectral index, sevoflurane, Fentanyl

\section{Introduction}

A successful GA is a reversible triad of analgesia, hypnosis and removal of reflex. We use many drugs to produce balanced anesthesia. An inadequate General anesthesia leads to intra operative awareness which may be with or without recall, while overdosage of drugs leads to delayed recovery and its possible postoperative complications. ${ }^{[1]}$

The administration of a minimum of 0.5 minimum alveolar concentration of the volatile agent will prevent awareness during GA. An EEG or AEP based monitors enables a reproducible, objective and continuous measurement of depth of anesthesia, even during the period when the patient is paralysed and all reflexes are abolished.BIS monitors consist of a soft sensor which has EEG electrodes producing a dimensionless number on the scale from 0-100 using its integrated custom software. This is used by the anaesthesiologist to decide about the level of GA using the BIS value as reference, based on it the anesthetic requirements are titrated. Systemic lidocaine infusion has been used in several studies to evaluate its benefits on the outcome of anesthesia. Having inflammation-modulatory properties, it significantly reduced pain and allowed more rapid discharge.$^{[2]}$ Similarly, studies have shown reduction in MAC value of inhalational agents by the intravenous lignocaine. Reduction in MAC value reduces their requirement. The effect of i.v.lidocaine on MAC was suggested to be due to its action at the spinal level by decreasing the motor response and its inhibitory effect on central nervous system. ${ }^{[3]}$ This study aims at evaluating the effect of intravenous lidocaine on sevoflurane requirements and monitoring the anesthetic depth by BI-SPECTRAL INDEX.

\section{Aim of Study}

The aim of this study is to know the effect of intravenous lignocaine on sevoflurane requirements as monitored by the bi-spectral index

\section{Materials and Method}

After obtaining institutional ethical committee approval and The informed written consent was obtained from the patients participating in the study was obtained. 60 ASA 1 and 2 patients of age 25 to 60 years undergoing elective laparoscopic surgeries expected to last for less than two hours were selected. Patients whose medical history, laboratory data, or physical examination showed evidence of abnormal hepatic or renal function or severe cardiovascular, pulmonary, neurological, psychiatric, or metabolic disease and allergy to local anesthetics were excluded from the study.

These patients were randomly allocated into two groups GROUP L, and GROUP S.

Pts baseline pulse rate, blood pressure and spo2 were noted preoperatively outside the operation theatre. No pre op anxiolytics were given to any patients. In the OT following monitors were attached. BIS monitor- pre induction BIS is noted.pulseoximeter, nibp,ecgcapnography- intraopIntraop oxygen concentration was also noted using oxygen analyser. Separate infusion pumps for either lignocaine or saline was prepared by separate staff blinded to the observing anesthesiologist was kept ready.After checking the anesthesia machine- (dragger work station with dragger vapourisers) and with all emergency drugs in hands. All 


\section{International Journal of Science and Research (IJSR) \\ ISSN (Online): 2319-7064}

Index Copernicus Value (2015): 78.96 Impact Factor (2015): 6.391

patients were pre medicated with injglycopyrollate $0.2 \mathrm{mg}$ i.m 15-30mins prior.

All patients were preoxygenated with $100 \%$ oxygen and induced with inj fentanyl $2 \mathrm{mcg} / \mathrm{kg}$ and injthiopentone $5 \mathrm{mg} / \mathrm{kg}$ or till BIS drops to 40 and intubation facilitated with injsuxamathonium $1.5 \mathrm{mg}-2 \mathrm{mg} / \mathrm{kg}$. and ET tube of size 8$8.5 \mathrm{~mm}$ for men and $7-7.5 \mathrm{~mm}$ for women were used. Intubation stress was attenuated with inj lignocaine $1.5 \mathrm{mg} / \mathrm{kg}$ of intravenous lignocaine in group L. Saline was used in group S.Following intubation anesthesia was maintained with N2O:O2 1:2 sevoflurane $2 \%$ and then titrated according to the BIS value to be kept between 40 60. Infusion of lignocaine for group $\mathrm{L}$ at a rate of $2 \mathrm{mg} / \mathrm{kg} / \mathrm{hr}$ started and in $\mathrm{S}$ group saline infusion started. After $10 \mathrm{mins}$ the flow rate decreased from $51 / \mathrm{min}$ to $31 / \mathrm{min}$.

Pt was put on ventilator volume controlled mode with tidal volume $7-10 \mathrm{ml} / \mathrm{kg}$, RR- $12-16$ according to ETCO2, PEEP3-5 cmH2O.

\section{Parameters monitored-}

Pulse rate, $\mathrm{BP}$, spo2, BIS, ETCO2Sevoflurane dial percentage is noted every 5 mins initially later every 15 mins.Additional doses of fentanyl given whenever the PR Or BP is $20 \%$ above the baseline with BIS between $40-60$ and ETCO2 Is between 30-35.

\section{Inclusion Criteria}

- ASA I \&II pts

- Age 18-60

- Both sex

\section{Exclusion Criteria}

- Age $<18,>60$,

- Patients with bmi less than 20 or more than 35 .

- Patients with history of reaction to lidocaine,

- Patients with history of seizures or using sedatives, hypnotics or any other drugs that affect bis.

\section{Statiscical Analysis}

The information collected regarding all the selected cases were recorded in a master chart. Data analysis was done with the help of computer using Epidemiological Information Package (EPI 2010)developed by Centre for Disease Control, Atlanta.Using this software range, frequencies, percentages, means, standard deviations, chi square, ' $t$ ' value and ' $\mathrm{p}$ ' values were calculated. ' $\mathrm{t}$ ' test was used to test the significance of difference between quantitative variables and Yate's and Fisher's chi square tests for qualitative variables. A 'p' value less than 0.05 is taken to denote significant relationship

\section{Results}

The two groups group ' $\mathrm{L}$ ' and ' $\mathrm{S}$ ' were comparable in the demographic data like age, sex, mean weight and they were comparable. Duration of surgery, type of procedure was also comparable showing no statistical difference. The flow rates were also same through out the procedure and intra operatively BIS value was maintained between 40-60 with average BIS value showing no difference.

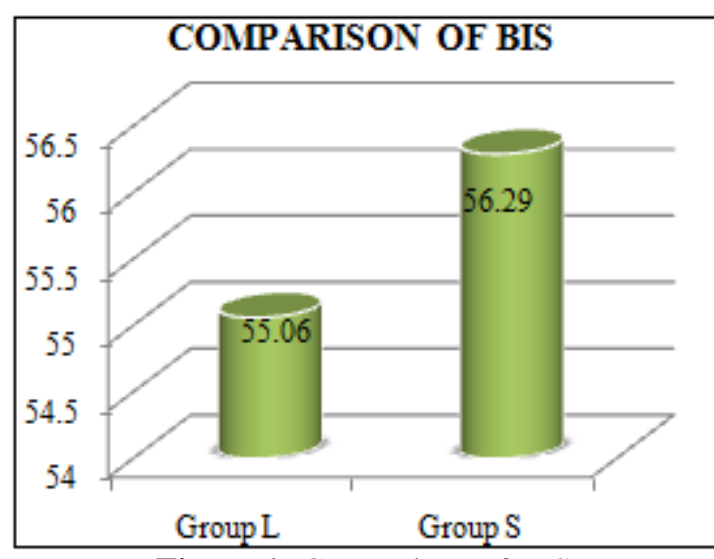

Figure 1: Comparison of BIS

The study infusion was blinded to the investigator and the heart rate blood pressure and saturation was also comparable showing that lignocaine infusion did not produce any hemodynamic stability.

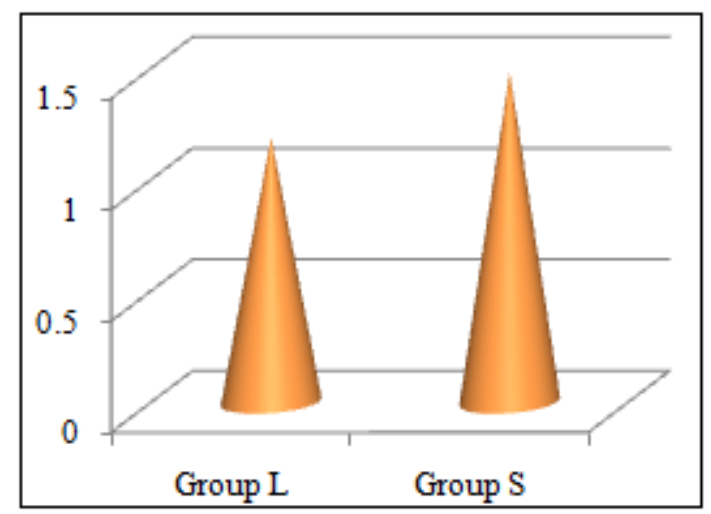

Figure 2: Comparision Dial Concentration

The dial concentration in lignocaine group needed to keep the BIS between 40-60 was significantly low leading on to the lower consumption of sevoflurane.

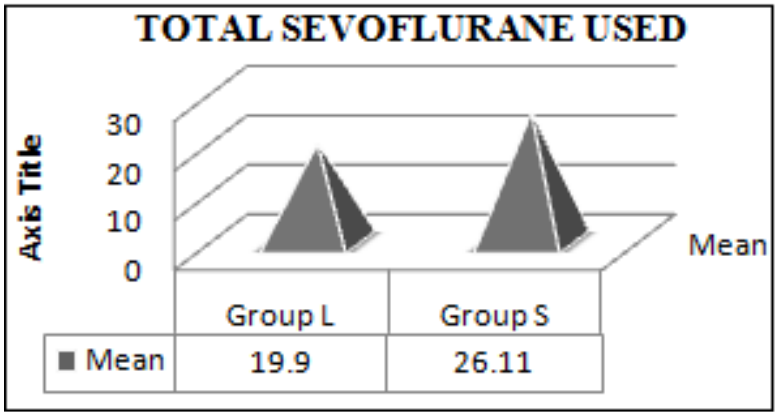

Figure 3: Total Sevoflurane Used

To standardize the consumption the flow rate was kept constant in both groups. The total sevoflurane as calculated by the DION'S formula was on a average $19.9 \mathrm{ml}$ in lignocaine group and it was $26.11 \mathrm{ml}$ in saline group showing drastic change in sevoflurane consumed. This reduction is about $24 \%$ reduction in sevoflurane consumption. This reduction on consumption produces cost effectiveness also.

The total fentanyl used intraoperative was also less in lignocaine group and producing significant difference. 


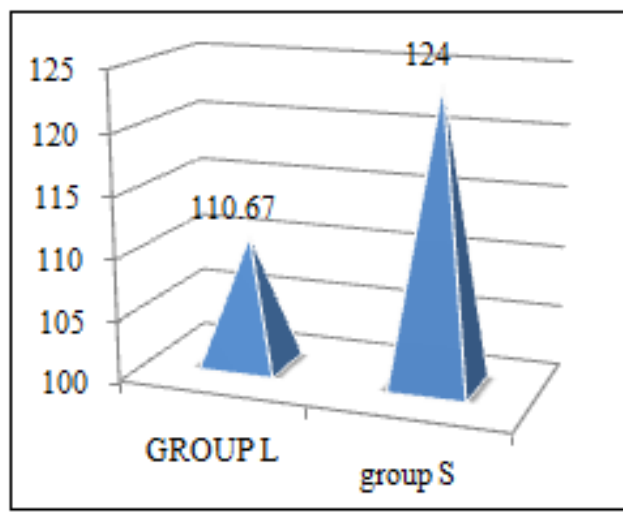

Figure 4: Comparison of Fentanyl

\section{Discussion}

Systemic lidocaine infusion has been used in several studies to know its benefits on the outcome of anesthesia. Having inflammatory-modulating property, it significantly reduces pain and allowes rapid discharge. In a metaanalysisof 8 randomized, controlled, clinical trials, patients who underwent abdominal surgeries while receiving continuous perioperative intravenous lignocaine, showed less duration of postoperative ileus, less pain, nausea, and vomiting and shorthospital stay. ${ }^{[4]}$ Hans pc et al. proved that patients who received perioperative lignocaine infusion, required less opioid and less post operative pain there by reducing the post operative ICU stay. ${ }^{[5]}$

Systemic lidocaine has proved to reduce MAC in animals. Hodson P S et al. observed in a study that lidocaine with or without ketamine significantly reduced the MAC of sevoflurane in dogs. It is also found that intravenous lidocaine decreased MAC of sevoflurane in anesthetized dogs without affecting blood pressure or heart rate.In our study the MAC reducing effect of sevoflurane and the hemodynamic stability of it with fentanyl requirements are monitored. ${ }^{[6]}$

DION'S FORMULA is used to calculate the sevoflurane used.

Amount of liquid sevoflurane used $=$ PFTM $/ 2412 \mathrm{~d}$

Where the variables represent

- P - Vaporizer dial concentration in percent

- F- Total fresh gas flow in liters/minute- 51/min

- T - Time for which the concentration $P$ was set in minutes

- M - Molecular mass of sevoflurane in grams - $200.055 \mathrm{mg}$

- d Density of liquid sevoflurane in grams/milliliter

- The fixed variables used were

- $\mathrm{d}$ (density of sevoflurane at $\left.21^{\circ} \mathrm{C}\right)=1.52 \mathrm{~g} / \mathrm{ml}$

- Substituting the fixed variables the equation can be rewritten as:

- Amount of liquid sevoflurane used $=0.2728 \mathrm{PT}(\mathrm{T}$ in mins)

Using this formula with the monitored parameters like flow rate, dial concentration and time, we have calculated the sevoflurane used totally for each case. The MAC reducing effect of lignocaine is its action on cerebral metabolic rate nd reduction of $\mathrm{CMRO} 2$ - cerebral oxygen demand. This reduces the anesthetic requirements. The effect of i.v. lidocaine on MAC was suggested to be due to its action at the spinal level by decreasing the motor response. The reduction in sevoflurane consumption is about $24 \%$ less with lignocaine group. This lignocaine infusion reduces the sevoflurane requirements there by reducing the total cost. $200 \mathrm{ml}$ of sevoflurane costs Rs. 2500. Using a cheaper drug with minimal side effects and better hemodynamic stability reduces the total cost of the anesthetic drugs.

\section{Conclusion}

Intravenous lignocaine in the form of bolus followed by infusion reduces the sevoflurane requirements by $24 \%$ to maintain the BIS level between 40-60 which is required to prevent intra op awareness and recall significantly maintaining the hemodynamic stability and also reduces the intra op analgesic requirements in patients undergoing laparoscopic surgeries.

\section{References}

[1] Hans GA, Lauwick SM, Kaba A, Bonhomme V, Struys MM, Hans PC, et alIntravenouslidocaine infusion reduces bispectral index-guided requirements of propofol only during surgical stimulation. Brit $\mathbf{J}$ Anaesth 2010;105:471-9.

[2] Lauwick S, Kim do J, Michelagnoli G, Mistraletti G, Feldman L, Fried G, CarliFIntraoperative infusion of lidocaine reduces postoperative fentanyl requirments in patients undergoing laparoscopic cholecystectomy. Can J Anaesth 2008;55:754-60

[3] Altermatt FR, Bugedo DA, Delfino AE, Solari S, Guerra I, Mun oz HR, et al.Evaluation of the effect of intravenous lidocaine on propofol requirements during total intravenous anaesthesia as measured by bispectral index. Brit J Anaesth 2012;108:979-83.Altermatt FR, Bugedo DA, Delfino AE, Solari S, Guerra I, Muñ oz $\mathrm{HR}$, et al.

[4] Meta-analysis of intravenous lidocaine and postoperative recovery after abdominal surgery. Brit $\mathrm{J}$ Surg 2008;95:1331-8. Marret E, Rolin M, Beaussier M, Bonnet F.

[5] Hans PC, etal.Intravenouslidocaine infusion reduces bispectral index-guided requirements of propofol only during surgical stimulation. Brit $\mathrm{J}$ Anaesth 2010;105:471-9.

[6] Hodgson PS, Liu SS Epidural lidocaine decreases sevoflurane requirement for adequate depth of anesthesia as measured by the bispectral index monitor. Anesthesiolgy 2001;94:799-803 